\title{
Die Rechtsverfassung der Wissensteilung
}

Thomas Gutmann"

Rezension zu Dan Wielsch, Zugangsregeln. Die Rechtsverfassung der Wissensteilung, Tübingen (Mohr Siebeck - Jus Privatum, Band 133), 2008, X, 303 Seiten, $84 €$, ISBN 978-3-16-149580-9

Die zu besprechende Studie von Wielsch ist die überarbeitete Fassung seiner an der Universität Frankfurt am Main entstandenen Habilitationsschrift. Sie entfaltet einen leitenden normativen Gedanken, die Begründung von Grenzen geistigen Eigentums. Subjektive Immaterialgüterrechte, so die These der Untersuchung, müssten systematisch um Regeln der erlaubnisfreien Nutzung ergänzt werden, damit die dezentrale Erzeugung von Wissen gewährleistet bleibe.

\section{Die epistemische Form der Bürgerlichen Gesellschaft}

Die Arbeit verknüpft in ihrem theoretischen Zugriff (vor allem in den $\mathbb{S} \mathbb{S}$ und 2, S. 12-116) informationsökonomische Überlegungen mit der Perspektive der Luhmann'schen Systemtheorie und verwendet als Brücke zwischen beiden Disziplinen den auf Friedrich v. Hayek zurückgehenden Gedanken der ,Wissensteilung'. Das Funktionieren von Märkten, so die Ausgangsthese der Untersuchung, hänge von Wissensstrukturen ab, die in modernen Gesellschaften „in transsubjektiven Prozessen sozialer Systeme“ generiert würden (S. 2). Der Wettbewerb sei als epistemischer Mechanismus zu begreifen, der davon zehre, dass das dezentrale, auf die einzelnen Marktakteure verteilte Wissen mobilisiert werden könne. Das Mittel hierzu sieht Wielsch mit Hayek in der rechtlichen Garantie subjektiver Eigentumsrechte und Vertragsfreiheiten, die dem einzelnen Wissensträger die Entscheidungskompetenz vermittelten, seine individuellen Fähigkeiten („knowledge, experience and skills“) selbst für die Auswahl der Verwendungsmöglichkeiten knapper Güter zu aktivieren. Diesen Zusammenhang - die Erzeugung und Sicherung von „Beobachtungsreichtum“ (S. 177, 191) - zu generalisieren ist das Anliegen Wielschs. Seine Studie will den rechtlichen Formen nachgehen, durch die „das auf psychische Systeme verteilte (dezentrale) Wissen für den Aufbau von Wissen in sozialen Systemen genutzt“ werden kann (S. 38, vgl. S. 3), insbesondere also der Frage, wie sich das in der Form von Immaterialgütern vorliegende kondensierte Wissen der Einzelnen für die Funktionserfüllung aller jeweils beteiligten sozialen Systeme instrumentalisieren lässt.

$\mathbb{} 1$ der Untersuchung, der der Rechtfertigung der Schaffung von Immaterialgütern und ihren Grenzen gilt, setzt mit einer ökonomischen Analyse ein, aus deren Perspektive die Verleihung von exklusiven individuellen Verfügungsrechten von vorneherein nur marktfunktional, d.h. als Instrument zur Organisation effizienten Wett-

* Prof. Dr. Thomas Gutmann lehrt Bürgerliches Recht und Rechtsphilosophie an der Westfälischen Wilhelms-Universität Münster. 
bewerbs begriffen wird. Wielsch zeigt in souveräner Verwendung der wirtschaftswissenschaftlichen Literatur, dass Immaterialgüter dem „informationsökonomischen Dilemma“ unterliegen: Während Schutzrechte notwendig scheinen, um dem Einzelnen Anreize zur Kreativität zu bieten, hemmen sie mit dem freien Zugang zu Informationsgütern zugleich die effiziente Nutzung des so erzeugten Wissens durch andere (sie „inhibieren positive Externalitäten“). Die sozialen Kosten der Unternutzung vorhandener Wissensbestände zu Lasten des Innovationswettbewerbs sprechen für Wielsch nachdrücklich für eine Beschränkung der von Immaterialgüterrechten bewirkten Zugangshemmnisse - eine „Logik der Perfektionierung von Schutzrechten ist aus der Sicht der Ökonomie nicht zu rechtfertigen“ (S. 30).

\section{Immaterialgüterrecht und Systemtheorie}

Der Autor unternimmt sodann eine Untersuchung des Immaterialgüterrechts aus systemtheoretischer Sicht (S. 31-81). Er setzt mit dem Befund der „Multifunktionalität" der geschützten Immaterialgüter ein, die neben ihrer Bedeutung für die Wirtschaft immer zugleich Kommunikationen in anderen Funktionssystemen (wie etwa der Wissenschaft, der Kunst oder der Politik) darstellten, deren jeweilige Sinnhorizonte für die einzelne schöpferische Leistung zudem konstitutiv seien. Eigentumsund zumal Immaterialgüterrechte beeinflussten deshalb die Möglichkeiten des Aufbaus von Wissen in verschiedenen sozialen Systemen gleichzeitig. Weil Immaterialgüter (die Wielsch von Konnotationen des Sacheigentums lösen möchte) in diesem Sinne soziale „Infrastrukturen“ darstellten (S. 110), sei „der Normgehalt des Art. 14 GG für die Wissensteilung in den Systemen verantwortlich“ (S. 80). Aus dieser Beobachtung leitet Wielsch vergleichsweise unvermittelt den „gesellschaftliche[n] Auftrag an das Recht“ ab, „Institutionen der Wissensteilung adäquat zu verfassen“ (S. 42), d.h. „umweltbewusste“ rechtliche Institutionen des Immaterialgüterrechts auszubilden, die den Operationsweisen und der Integrität aller betroffenen systemische Prozesse der Wissensteilung gleichermaßen gerecht werden. Rechtstheoretisch und grundrechtsdogmatisch führt dieser („recht anspruchsvolle“, S. 81) Auftrag zu einer weitreichenden „funktionellen Perspektivierung subjektiver Eigentumsrechte“: „Der Schutz subjektiver Rechte dient dem Schutz transindividueller Strukturen, auf die der Einzelne [...] für sein eigenes autonomes Handeln angewiesen ist“ (S. 47, 271) - der „Schutz des Einzelnen [wird] umgelenkt auf den Schutz von den für die Autonomie von Systemen grundlegenden Institutionen der Wissensteilung “ (S. 10).

Mit der Ausnahme des Kunstsystems, in dem nach Wielschs Ansicht die für die ästhetische Kommunikation notwendigen Bedingungen regelmäßig gerade mit Hilfe der exklusiven Zuordnung von rechtlicher, Werkherrschaft' an den Produzenten gewährleistet werden können (S. 44), verlangen, wie die Arbeit im Folgenden zeigt, der Schutz transindividueller systemischer Strukturen und die Aufgabe des Rechts, die „Anreicherung kollektiver Wissensbestände“ zu garantieren, grundsätzlich eine sys- 
tematische Beschränkung subjektiver Immaterialgüterrechte durch die „Gewährleistung von Nutzerfreiheiten in einem Umfang, der die Möglichkeiten der dezentralen Erzeugung von Wissen durch eine Vielzahl von Akteuren erhält" (S. 6).

Als theoretischen Überbegriff für die unterschiedlichen rechtlichen Konstruktionsmöglichkeiten, derer sich diese Aufgabe der Abstimmung von Ausschließlichkeitsrecht und Nutzungsfreiheit bedienen kann, verwendet Wielsch anstelle der herkömmlichen Terminologie (Schutzbereichs- und Schrankenbestimmungen) den Begriff der ,Zugangsregeln', wobei ,Zugang' nichts anderes meint als das „Fehlen einer exklusiven Zuordnung von Handlungsbefugnissen aufgrund Einschränkung des Verbotsrechts“, rechtstheoretisch also die für einen Nutzer bestehende Erlaubnis zur Vornahme einer immaterialgutsbezogenen Handlung (S.64f.). Die Begriffswahl rechtfertigt der Autor mit dem Umstand, dass die von ihm diskutierten Zugangsregeln nicht auf die Unterscheidung Individual- vs. Allgemeininteresse abstellten, sondern mit der komplexeren Referenz auf soziale Systeme operierten.

In einem zentralen Abschnitt zur „Verfassung als Koordinationsrecht“ (S. 66 f.) überführt Wielsch seine systemtheoretisch inspirierten Reflexionen auf rechtlichen Grund. Mit der (auf die frühe und werkgeschichtlich nicht unproblematische Schrift Luhmanns über „Grundrechte als Institution“ abstellenden) These, dass die unterschiedlichen systemischen Rationalitäten „,in den Grundrechten verankert“ seien und die operative Integrität sozialer Systeme nicht zuletzt über grundrechtlich garantierte Freiheiten abgestützt werde (S. 66 f., vgl. S. 34), reformuliert er seine These nun grundrechtsdogmatisch. Wielsch versucht dabei zu plausibilisieren, dass hinter dem von ihm in Anspruch genommenen Prinzip „der Gleichrangigkeit von Ausschlussprinzip und Nutzerfreiheit ein gleichrangiges Nebeneinander der Grundrechte von Eigentums- und Wettbewerbsfreiheit steht“ (S. 70), das durch Art. 14 Abs. 2 GG als „Kompatibilisierungsnorm“ und eine systemtheoretisch informierte Konzeption der dort postulierten Bindung des Eigentums an das Allgemeinwohl zum Ausgleich gebracht werden könne.

Wielschs für die Arbeit zentrale Doppelthese zur „Ermöglichung grundrechtlich geschützter Freiheit durch die Inpflichtnahme eines anderen Privaten“ (S. 79) gründet wiederum in der fragilen Prämisse der wechselseitigen Übersetzbarkeit von Systemtheorie und Verfassungsrecht: Grundrechtsdogmatisch lasse sich das Gebot des Art. 14 Abs. 2 GG und mit ihm die Sozialbindung geistigen Eigentums dort aktivieren, wo die Nichteigentümer zur Realisierung ihrer Grundrechte auf den Zugang zum Immaterialgut „angewiesen“ seien und dies die Schutzgebotsfunktion ihrer Grundrechte auslöse; weil die Handlungs- und Kommunikationsmöglichkeiten der Einzelnen aber jeweils systemisch konstituiert seien, liege „die Bejahung eines Angewiesenheitsverhältnisses insbesondere dort nahe, wo die Funktionsfähigkeit zentraler Institutionen der Wissensteilung im jeweiligen System gefährdet ist“" (S. 75). 
Art. 14 Abs. 2 GG wird so zur universellen „'Sollbruchstelle' für die Rezeption der verschiedensten systemischen Rationalitäten“ (S. 81) - die Logik der Systeme bedient sich listig der Grundrechte der Einzelnen.

\$2 der Untersuchung (S. 82-116) gilt sodann den „Institutionen der Wissensteilung“ und vertieft, in dem das Werk insgesamt kennzeichnenden spiralförmig verlaufenden Gang der Darstellung, die bereits in Anspruch genommene Begrifflichkeit. In einer beeindruckenden, wissens- und organisationssoziologisch informierten Darstellung fundiert Wielsch sein Konzept der Institution und analysiert Markt, Unternehmen (sowie später, S. 192 ff., in innovativer Weise auch das „Netzwerk“) als Institutionen der Wissensteilung, in denen Beobachtungskapazität und Selektionsautorität auf rechtlich je eigentümliche Weise zur kollektiven Erzeugung von Wissen verfasst sind.

\section{Dogmatische Umsetzung}

Die „anwendungsorientierten“ $\mathbb{S} \mathbb{S} 3$ bis 5 der Arbeit gelten sodann einer differenzierten rechtsdogmatischen Umsetzung des skizzierten Programms, die im Rahmen dieser Rezension nur unzureichend gewürdigt werden kann. Wielsch geht nun systematisch der Bedeutung von Zugangsregeln für den Schutz von Institutionen auf verschiedenen Rechtsgebieten nach. Der umfangreiche $\mathbb{} 3$ („Wettbewerbsrechtliche Zugangsregeln“, S. 117-219) dient einer Untersuchung der Voraussetzungen, unter denen Dritten die Freiheit zur Mitnutzung von Schutzgegenständen aus Gründen des Wettbewerbsrechts gewährt werden kann. Er arbeitet, mit Seitenblicken auf das USamerikanische Kartellrecht, insbesondere anhand einer kritischen Analyse der Rechtsprechung des EuGH zum Immaterialgüterkartellrecht heraus, dass und in welcher Weise wettbewerbsspezifische Zugangsregeln aus Art. 82 EG - also Zwangslizenzen und ggf. auch Offenlegungs- und Informationspflichten - im Falle missbräuchlicher Zugangsverweigerung das Handeln marktbeherrschender Schutzrechtsinhaber auf den (verbundenen) Märkten der Informationsindustrie begrenzen.

Wielschs eigene, ,systemfunktionale' normative Rekonstruktion leitet sich konsequent aus seinem theoretischen Ansatz ab: Gerade weil der Wettbewerb um Immaterialgüter als Verfahren zur dezentralen Innovation expliziten Wissens in besonderer Weise von ausreichenden Möglichkeiten zur freien Nutzung dieser Güter abhänge und Wettbewerber hier regelmäßig auf die Mitnutzung von normativ geschützten oder faktisch kontrollierten Immaterialgütern angewiesen seien, müssten solche „Bottleneck-Ressourcen“ letztlich als „funktionale Gemeinschaftsgüter“ konstruiert werden. Mit starken Gründen schlägt Wielsch zu diesem Zweck eine Auslegung des Art. 82 EG vor, die sich an der essential facilities doctrine des angelsächsischen Wettbewerbsrechts orientiert (S. $186 \mathrm{f}$.).

Im folgenden $\mathbb{4}$ ( „Netzwerk als Institution der Wissensteilung“, S. 193-233) wendet sich Wielsch sodann einem Gegenstand zu, dem als „Avantgarde“ (S.213, vgl. 
S. 211) der Ko-Evolution von technologischen, organisatorischen und rechtlichen Strukturen ersichtlich seine Sympathie gilt. Er zeigt hier, wie sich die Entwicklung von Open Source Software (wie etwa des „Linux“-Systems) und die ihr zugrunde liegende Form einer generalisierten Reziprozität zwischen einer unbestimmten Vielzahl von partizipierenden Personen rechtlich verfassen lässt. Wielsch analysiert, wie sich solche Netzwerke privatautonom gestalteter Lizenzmodelle bedienen, um Schutzrechte zugunsten der Nutzer netzwerkspezifisch umzupolen, und wie diese privatrechtliche Konstruktion freier Software als öffentliches Gut (insbesondere die im Rahmen der GNU General Public License verwendete sog. „copyleft“-Klausel, Ziffer 2 lit. b GPL), wenngleich nicht ohne Spannungen, in das deutsche Privat- und Urheberrecht eingepasst werden kann.

$\$ 5$ der Studie (S. 234-266) zeigt sodann, dass die Funktion des Internets als (Massen-) Medium dezentraler Wissensteilung nur durch medienspezifische Zugangsregeln gewährleistet werden kann. Wielsch weist hier insbesondere auf, dass es die durch das sog. „End-to-End Principle“ (e2e-Prinzip) vermittelte physikalische Internet-Architektur ist, die als technologische Zugangsregel die diskriminierungsfreie Neutralität des Netzwerks und seinen Charakter als Gemeinschaftsgut sichert, und schlägt zur Normierung seiner Unverfügbarkeit eine Orientierung an der Public Trust Doctrine des amerikanischen Umweltrechts vor (S. 253). Zwei weitere, an sich nicht notwendige, aber instruktive Abschnitte gelten einer nutzerfreundlichen Rekonstruktion der urheberrechtlichen Rechtslage in Bezug auf das sog. Hyperlinking und auf die Suche im Netz, bevor der Autor im abschließenden $\$ 6$ (S. 267-284) die Prämissen und Ergebnisse seiner Untersuchung als „Elemente einer Rechtsverfassung der Wissensteilung" nochmals zusammenfasst.

Das Werk vermag in mehrerer Hinsicht zu beeindrucken. Sein normatives Anliegen ist klar und gut begründet. Der Zugriff des Autors auf die (deutsche, europäische und amerikanische) Rechtsdogmatik sowie die verwendeten Theoriebestände der Ökonomie und der Soziologie ist gleichermaßen souverän, und der Text ist jedenfalls für denjenigen ein intellektuelles Vergnügen, der bereit ist, sich auf seine Sprache einzulassen. Vor allem aber gelingt es Wielsch auf herausragende Weise, seinen Gegenstand von einer hinreichend komplexen, aber einheitlichen normativen Leitidee her zu organisieren und auf diese Weise zu demonstrieren, warum eine rechtstheoretisch angeleitete Rechtsdogmatik die bessere Rechtsdogmatik sein kann. Zudem ist seine Argumentation gleichsam doppelt genäht: Das Buch lässt sich, unter Ausblendung der systemtheoretischen Begrifflichkeit, auch schlicht als verfassungsrechtlich eingekleidetes normatives Plädoyer für ein innovationsfreundliches und insoweit gemeinwohlorientiertes Immaterialgüterrecht lesen ${ }^{1}$ - wobei auch weniger theorieaf-

1 Damit ist Wielsch nicht allein, vgl. z.B. W. Kingston, Beyond Intellectual Property. Matching Information Protection to Innovation, Cheltenham Glos 2010. 
fine Juristen „alter Schule“ nicht umhin kommen werden, einzuräumen, dass mit den verwendeten informationsökonomischen und systemtheoretischen Instrumenten besser und genauer beschrieben und erklärt werden kann, worin genau die kollektiven Güter bestehen, um derentwillen der Schutz geistigen Eigentums beschränkt werden sollte. Insgesamt also: Eine starke Stimme, ein wichtiges Buch.

Im Folgenden soll nun aber in einer knappen Skizze nach dem spezifischen Mehrwert gefragt werden, den der Rekurs Wielschs auf die Systemtheorie für seine über den eigentlichen Gegenstand der Arbeit hinausweisende normative Argumentation tatsächlich bieten kann.

\section{Systemtheoretisch gerechtes Recht?}

Die Theorie selbstreferentieller Systeme dürfte hinsichtlich ihrer Abstraktionshöhe und ihres einheitlichen theoretischen Zugriffs auf die soziale Welt gegenwärtig ohne Alternativen sein. Ihr Potential für die Rechtswissenschaft erscheint bei weitem nicht ausgeschöpft ${ }^{2}$, und die unter deutschen Juristen bisweilen erhobene grundsätzliche Kritik an ihr ist meist ebenso grundsätzlich verfehlt. ${ }^{3}$ Wielschs Zugriff gibt jedoch Anlass für einige Rückfragen.

(1) Fragen hinsichtlich der Art und Weise der Wielsch'schen Verwendung der Systemtheorie ergeben sich nicht in erster Linie wegen seiner scheinbar handlungstheoretischen Fundierung des Systembegriffs („Aus freiheitlichem Handeln der Einzelnen entstehen kollektive Ordnungen, [...] die hier als Systeme bezeichnet werden “, S. 67, vgl. S. 107) oder wegen des Umstands, dass sich der von ihm in Anspruch genommene Begriff des ,Wissens' keinesfalls bruchlos in das Luhmann'sche Konzept systemspezifischer Kommunikationen rückübersetzen lässt. Im Vordergrund steht vielmehr seine von Gunther Teubner ${ }^{4}$ entlehnte Leitidee des Rechts als eines ,intersystemischen Kollisionrechts' (Teubner) bzw. eines „multilateralen Konstitutionalismus“ (Wielsch), in der das Recht „ein der Gesellschaft gerechtes Recht“ ist (S. 5), weil und insoweit es als „,iustitia mediatrix“ die Funktion einer Beschützerin der systemischen

2 Vgl. zu einer operativ-funktionalistischen Theorie der Rechtsvergleichung jüngst B. Fateh-Moghadam, Die Einwilligung in die Lebendorganspende. Die Entfaltung des Paternalismusproblems im Horizont differenter Rechtsordnungen am Beispiel Deutschlands und Englands, München 2008.

3 Vgl. exemplarisch O. Lepsius, Steuerungsdiskussion, Systemtheorie und Parlamentarismuskritik, Tübingen 1999, der sich zu der These versteigt, die Modellannahmen der Systemtheorie seien „weder deskriptiv noch normativ mit einer grundgesetzlich orientierten Rechtswissenschaft kompatibel " und ihre Schlüsse verstießen gegen „das Menschenbild des Grundgesetzes“, ja gar gegen Art. 1 Abs. 1 GG (S. 71, 54 f.). Ebenso wenig Sinn ergibt es, der Theorie der Autopoiesis „rechtsethischen Nihilismus“ vorzuwerfen, so aber D. von der Pfordten, Rechtsethik, München 2001, S. $119 \mathrm{ff}$.

4 G. Teubner, Recht als autopoietisches System, Frankfurt a. Main 1989, S. 123 ff.; ders., Altera Pars Audiatur: Das Recht in der Kollision anderer Universalitätsansprüche, ARSP, Beiheft 65 (1996), 199-220 (204ff.); ders., Dreiers Luhmann, in: R. Alexy (Hrsg.), Integratives Verstehen: Die Rechtsphilosophie Ralf Dreiers, Tübingen 2005, 199-211 (201f.) und ders., Die anonyme Matrix: Zu Menschenrechtsverletzungen durch ,private‘ transnationale Akteure, Der Staat 45 (2006), 161-187 (169). 
Eigenlogiken und einer Vermittlerin zwischen diesen (sowie zwischen diesen und seiner eigenen Logik) übernimmt. ${ }^{5}$

Luhmanns Konzeption selbstreferentieller Systeme ${ }^{6}$ thematisiert den historischen Prozess und gegenwärtigen Stand der Ausdifferenzierung gesellschaftlicher Teilsysteme und deren Umstellung auf eine jeweils eigene Rationalität. Jedes Teilsystem orientiert sich an einer Leitdifferenz, mit Hilfe derer es sich von seiner Umwelt abgrenzt und so seine Identität in einem ständigen, nicht zielgerichteten Prozess autopoietischer Selbstreproduktion aufrecht erhält, der aus Ereignissen sinnhafter Kommunikation ${ }^{7}$ besteht. Weil Systeme ihre Abgrenzung zur Umwelt und damit ihre Einheit, also die Regeln, nach denen sie operieren und die Elemente, aus denen sie bestehen, selbst (autopoietisch) erzeugen müssen, können sie zwar ihre Umwelt beobachten, sind dabei aber ausnahmslos „operativ geschlossen “, oder, was das gleiche meint, autonom. ${ }^{8}$ Deshalb beharrt Luhmann mit guten Gründen darauf, dass sich Gesellschaft nur mehr polykontextural ${ }^{9}$ beschreiben und nicht mehr als Ganzes im Ganzen repräsentieren lässt. Es kann, wie Luhmann betont, „keine gesellschaftliche Rangordnung der Teilsysteme im Verhältnis zueinander geben, sondern die Einwirkungen der Teilsysteme aufeinander [...] wechseln gesellschaftlich unsteuerbar von Situation zu Situation. " 10 Es ist eine der Pointen der Theorie selbstreferentieller Systeme, dass auch das Recht nicht die Zentralperspektive der Gesellschaft usurpieren kann, weil es dazu seine autopoietische Struktur, und damit sich selbst aufgeben müsste. ${ }^{11}$

Wielsch sieht dies selbst, ${ }^{12}$ beharrt aber dessen ungeachtet darauf, dass unter den gesellschaftlichen Funktionssystemen das Recht eine Sonderstellung einnimmt, weil es „spezialisiert [ist] auf die intersystemischen Beziehungen, es steht bildlich gleichsam ,inmitten' der Systeme und ihrer Umwelten, im ,Zwischenraum ““. ${ }^{13}$ Das damit ausgesprochene Programm eines Rechts, das „der Funktionsweise anderer Systeme ,gerecht" wird“, indem es sich an dem Zweck des „Schutzes der Adaptions- und Evolutionsfähigkeit von sozialen Systemen ausrichtet" ${ }^{* 14}$ und damit deren Binnen-

5 Vgl. hierzu auch D. Wielsch, Die epistemische Analyse des Rechts. Von der ökonomischen zur ökologischen Rationalität in der Rechtswissenschaft, JZ 2009, 67-77 und ders., Iustitia mediatrix: Zur Methode einer soziologischen Jurisprudenz, in: G.-P. Calliess et al. (Hrsg.), Soziologische Jurisprudenz. Festschrift für Gunther Teubner zum 65. Geburtstag, Berlin 2009, 395-414.

6 N. Luhmann, Soziale Systeme. Grundriß einer allgemeinen Theorie, Frankfurt a. Main 1984.

7 N. Luhmann, Das Recht der Gesellschaft, Frankfurt a. Main 1993, S. 35.

8 Lubmann, Recht der Gesellschaft (Fn. 7), S. 44, 62.

9 Lubmann, Gibt es in unserer Gesellschaft noch unverzichtbare Normen?, Heidelberg 1993, S. 7; vgl. ders., Ökologische Kommunikation, Opladen, 3. Auflage 1990, S. 216.

10 Luhmann, Unverzichtbare Normen (Fn. 9), 16.

11 Vgl. Lubmann, Recht der Gesellschaft (Fn. 7), S. $154 \mathrm{f}$.

12 Wielsch, Epistemische Analyse (Fn.5), S. 70.

13 Ebd., S. 73.

14 Ebd., 69. 
perspektiven mit übernimmt, überdehnt die Grenzen dessen, was die „rechtseigene Rationalität“ (Wielsch) leisten kann. Auch in der Luhmann'schen Perspektive, derzufolge „das System seine Einwirkungen auf die Umwelt an den Rückwirkungen auf es selbst kontrollieren muss, wenn es sich rational verhalten will“, ${ }^{15}$ bleibt es vielmehr dabei, dass wie „jedes System [...] auch das Recht nur nach Maßgabe eigener Strukturen resonanzfähig “ ist. ${ }^{16}$ Die Vorstellung des Rechts als soziales Muttersystem, das die anderen Systeme „ökologisch“ am Busen nährt und zwischen den Kindern mit gerechter Hand ausgleicht und vermittelt, schmeichelt dem Juristen, ist aber mit den Mitteln der Luhmann'schen Theorie autopoietischer Systeme schwerlich darstellbar. Dies alles legt die Vermutung nahe, dass das von Wielsch intendierte „ökologische Recht“, das die ungesteuert evolutionär verlaufenden autopoietischen Prozesse rechtlich in die Regie nehmen soll, weitaus stärkere Umbauarbeiten an der Architektur der Systemtheorie voraussetzt als Wielsch dies ausweist.

Hinzu kommt, dass das Werk eine Lesart zumindest nahe legt, nach der Wielsch vorschlägt, die dem Recht eigentümliche Recht/Unrecht-Unterscheidung durch ein rechtsfremdes Maximierungsprinzip zu überlagern. Mit dem Metaziel einer „optimierte[n] gesellschaftliche[n] Nutzung von Wissen und Information“ (S. 17) im Interesse des „wirtschaftlichen Erfolg[s] der bürgerlichen Gesellschaft“ (S. 1) ist die Leitperspektive der Untersuchung dann ohnehin eine (wissens-) ökonomische und damit auf die spezifische Reflexionsform des Wirtschaftssystems bezogen. Konsequent kritisiert der Autor herkömmliche Spielarten der ökonomischen Analyse des Rechts auch nur wegen ihres nicht systemtheoretisch vermittelten und insoweit naiven Zugriffs (etwa S. 191). Das Buch bietet in dieser Lesart letztlich ordoliberalism reloaded, eine Rekonstruktion der Rechtsordnung aus dem Geist des wirtschaftsfunktionalen Kartellrechts, und insoweit gerade keinen systemtheoretischen Begriff des Rechts.

(2) Luhmanns Theorie ist second order observation, genauer: eine Beobachtung dritter Ordnung, die dem Rechtsystem dabei zusieht, wie sich dieses selbst beobachtet, selbst beschreibt und sich selbst fortlaufend rekonstituiert. Die Systemtheorie des Rechts ist deshalb eine Fremdbeschreibung ${ }^{17}$ des Rechtssystems. Im Gegensatz zu theoretischen Reflexionsformen des Rechts, die selbst normative Geltungsansprüche erheben und deshalb dem Rechtsystem zurechenbar sind, will und muss die Luhmann'sche Systemtheorie als solche normative Implikate ganz ausdrücklich vermeiden. ${ }^{18}$ Die systemische „Autopoiesis ist kein politisches und auch kein ethisches Kriterium der Akzeptabilität von Recht.“19

15 Luhmann, Soziale Systeme (Fn.6), S. 642.

16 Luhmann, Ökologische Kommunikation (Fn.9), S. 133.

17 Lubmann, Recht der Gesellschaft (Fn.7), S. 16 f., 547, vgl. S. 24, 497.

18 Ebd., S. 31.

19 Ebd., S. 83. 
Für das Recht selbst - und damit für die Rechtstheorie im engeren Sinn, die herausbekommen will, womit es das Recht nach seinem eigenen Verständnis zu tun hat gilt hingegen auch aus systemtheoretischer Sicht, dass Normen sich weder aus Fakten ableiten noch auch nur als Fakten beschreiben lassen, wenn man ihrem spezifischen Sinn und Forderungscharakter gerecht werden will. „Für die Rechtswissenschaft geht es um eine normative Ordnung “. ${ }^{20}$ Die Entscheidung darüber, welche Fakten, Befunde oder sonstigen systemexternen Irritationen für das Recht von Relevanz sind, kann deshalb nur eine rechtliche, also normative Entscheidung sein. ${ }^{21}$ Die Systemtheorie liefert mithin selbst die Begründung dafür, warum ihre Beobachtungen als solche keine normativen Implikationen für den Diskurs des Rechts besitzen können. ${ }^{22}$ Es gibt kein systemtheoretisch „richtiges Recht“, deshalb können „systemtheoretisch hergeleitete" normative Setzungen nichts anderes sein als normative Fehlschlüsse oder aber bloße Dezision.

Ähnlich wie bereits Gunther Teubner, der für seine Theorie des Rechts als autopoietisches System einen „normativ-analytischen Doppelstatus“ reklamierte und dem Recht kurzerhand empfahl, aus der systemtheoretischen Beschreibung seiner Operationsweise ,in seiner Entscheidungspraxis operative Konsequenzen [zu] ziehen “23, geht auch Wielsch von der Beobachtung umstandslos in die Präskription über und will dem Leser mit systemtheoretischen Mitteln nahebringen, wie „richtiges“ bzw. „gerechtes Recht“ auszusehen hat. Weil das nicht begründet möglich ist, kreist Wielschs normativ gewendete Systemtheorie um eine Leerstelle. Ihrer scheinbaren systemtheoretischen Hüllen entkleidet, müssen die normativen Thesen, (a) dem Recht solle es primär um den Schutz transindividueller Strukturen gehen und (b) die Rechtswissenschaft solle ihre „Fixierung [...] auf das subjektive Recht und die Person als dessen Träger" (S.269 und passim) auch deshalb überwinden, weil (c) aus der konstitutiven Funktion transindividueller Strukturen für individuelles Handeln auch der normative Primat ersterer vor letzterem folge, vielmehr normativ begründet werden.

Für das Unternehmen, den Einzelnen nicht nur zum Diener zweier Herrn, sondern vieler Systeme zu machen, steht ein breites Angebot an nichtliberalen Theoriebausteinen zur Auswahl (wobei Wielsch keinen Zweifel daran lässt, dass er insoweit zumindest nicht auf das regressive, vormoderne Modell einer immanenten Pflichtbindung subjektiver Rechte rekurrieren will, S. 60). Gerade der systemtheoretisch informierte Autor wird hierbei jedoch nach der Anschlussfähigkeit seiner Thesen an einen Rechtsdiskurs fragen müssen, der auch von Verfassungs wegen mit guten

20 Ebd., S. 14; vgl. ebd., 12, 33, 502 und ders., Unverzichtbare Normen? (Fn.9), S. 5.

21 Luhmann, Recht der Gesellschaft (Fn.7), S. 87.

22 Ebd., 565; vgl. ders., Unverzichtbare Normen? (Fn.9), S. 4.

23 Teubner, Recht als autopoietisches System (Fn.4), S. 86, 29. 
Gründen durch eben jenen starken normativen Individualismus geprägt ist, den Wielsch im Wesentlichen verabschieden will. Wielsch wird hierbei nicht zuletzt auch die massiven paternalistischen Implikationen seines Ansatzes zu legitimieren haben: Da für ihn der „personale Träger des Eigentumsrechts, der [...] selbst zum Schnittpunkt unterschiedlicher Systeme wird, [...] regelmäßig überfordert ist, die verschiedenen Perspektiven - seine ,Rollen' in der modernen, funktional differenzierten Gesellschaft - zu integrieren“, muss das objektive Recht solche Intersystemkonflikte entscheiden und z.B. mit der Autonomie der Kunst auch den Künstler vor sich selbst schützen, falls diesem einfallen sollte, mehr als ökonomischer „Verwerter“ seiner Fähigkeiten auf dem Kunstmarkt denn als Künstler zu agieren: „Das Schutzrecht wird dann zum Durchsetzungsinstrument einer bestimmten Systemlogik zu Lasten einer anderen“ (S. 69), wobei es offenbar nicht mehr der überforderte Grundrechtsträger selbst sein soll, der entscheiden darf, welcher Systemlogik er folgen möchte.

Den Einzelnen ist jedenfalls zu raten, sich für eine Reise durch Wielschs Welt des „ökologischen“ Rechts warm anzuziehen. Es weht ein kalter Wind zwischen den Systemen. Dieser Befund will und kann die wissenschaftliche Bedeutung des rezensierten Werks allerdings nicht mindern. 